Mon. Not. R. Astron. Soc. 000, 000-000 (0000) Printed 7 October $2018 \quad$ (MN LATEX style file v2.2)

\title{
Galaxy Merger Statistics and Inferred Bulge-to-Disk Ratios in Cosmological SPH Simulations
}

\author{
Ariyeh H. Maller ${ }^{1,2}$, Neal Katz ${ }^{1}$, Dušan Kereš ${ }^{1}$, Romeel Davé ${ }^{3}$, David H. Weinberg ${ }^{4}$ \\ ${ }^{1}$ Astronomy Department, University of Massachusetts Amherst, 710 N. Pleasant St., Amherst, MA 01003 \\ ${ }^{2}$ Department of Physical and Biological Sciences, New York City College of Technology, 300 Jay St., Brooklyn, NY 11201 \\ ${ }^{3}$ University of Arizona, Steward Observatory, 933 North Cherry Avenue, Tucson, AZ 85721 \\ ${ }^{4}$ Department of Astronomy, Ohio State University, 140 West 18th Avenue, Columbus, OH 43210
}

7 October 2018

\begin{abstract}
We construct merger trees for galaxies identified in a cosmological hydrodynamical simulation and use them to characterize predicted merger rates as a function of redshift, galaxy mass, and merger mass ratio. At $z=0.3$, we find a mean rate of 0.054 mergers per galaxy per Gyr above a 1:2 mass ratio threshold for massive galaxies (baryonic mass above $6.4 \times 10^{10} M_{\odot}$ ), but only $0.018 \mathrm{Gyr}^{-1}$ for lower mass galaxies. The mass ratio distribution is $\propto R_{\text {merg }}^{-1.2}$ for the massive galaxy sample, so high mass mergers dominate the total merger growth rate. The predicted rates increase rapidly with increasing redshift, and they agree reasonably well with observational estimates. A substantial fraction of galaxies do not experience any resolved mergers during the course of the simulation, and even for the high mass sample only $50 \%$ of galaxies experience a greater than $1: 4$ merger since $z=1$. Typical galaxies thus have fairly quiescent merger histories.

We assign bulge-to-disk ratios to simulated galaxies by assuming that mergers above a mass ratio threshold $R_{\text {major }}$ convert stellar disks into spheroids. With $R_{\text {major }}$ of 1 : 4, we obtain a fairly good match to the observed dependence of early-type fraction on galaxy mass. However, the predicted fraction of truly bulge-dominated systems $\left(f_{\text {bulge }}>0.8\right)$ is small, and producing a substantial population of bulgedominated galaxies may require a mechanism that shuts off gas accretion at late times and/or additional processes (besides major mergers) for producing bulges.
\end{abstract}

Key words: galaxies:formation — galaxies:spiral - galaxies:elliptical

\section{INTRODUCTION}

It has long been suggested that the spheroidal components of galaxies are a result of galaxy mergers (e.g., Toomre \& Toomre 1972), and that a galaxy's merger history therefore plays a central role in determining its Hubble type. Numerical simulations of disk galaxy mergers produce systems with many of the properties of observed elliptical galaxies (e.g., Barnes 1988; Hernquist 1992; Barnes \& Hernquist 1996; Naab et al. 1999; Springel 2000; Bendo \& Barnes 2000; Cretton et al.|2001; Naab \& Burkert 2003; Cox et al. 2004; Springel \& Hernquist 2004), including the telltale low surface brightness shells and tidal features that are often revealed by deep imaging (e.g., Malin \& Carter 1983; Schweizer et al. 1990; van Dokkum 2005). However, present simulations do not have the dynamic range needed to track the morphological evolution of individual galaxies while simultaneously modeling representative cosmological volumes, so it is unclear whether the merger hypothesis can explain the observed frequency and environment dependence of Hubble types. Semi-analytic models of galaxy formation have provided the main avenue for progress on this question, using simplified recipes to convert disk material in stellar bulges following mergers (e.g. Kauffmann et al. 1993; Kauffmann 1996; Baugh et al. 1996; Somerville \& Primack 1999; Cole et al. 2000; Bell et al. 2003a; Hatton et al. 2003; Nagashima \& Yoshii 2004). These models have achieved reasonable success in reproducing observed galaxy distributions, but they rest on an approximate treatment of dynamical friction to compute galaxy merger rates from dark halo merger rates, in addition to the assumptions of the merger recipes themselves. One recent semi-analytic study that tracks "galaxies" as sub-halos in high resolution N-body simulations yields results significantly different from those of other models (Kang et al. 2004). In addition, the effects of dynamical friction and tidal disruption on sub-halos will be altered by the presence of tightly bound gas and stars within them.

In this paper, we compute the merger histories of galax- 
ies formed in a smoothed particle hydrodynamics (SPH) cosmological simulation. While this simulation does not have the resolution required for reliable morphological analysis, it incorporates all of the physics that should have a major effect on merger rates, and we can apply semi-analytic style recipes to compute resulting distributions of Hubble types. We can also address, at least in part, the related issues of survival and angular momentum of galaxy disks.

Stellar disks are fragile, and one important question for cosmological models is whether they predict a low enough merger rates to preserve thin disks (Toth \& Ostriker 1992). In addition, disk galaxies forming in cosmological SPH simulations frequently lose angular momentum in late-time merger events, and the simulated galaxies have, on average, less angular momentum than observed disks (Navarro \& Steinmetz 2000). Recent simulations have produced some examples of disk galaxies with realistic angular momentum properties (Governato et al. 2004; Robertson et al. 2004), but these results are strongly dependant on the galaxy's merger history. Therefore, without knowing the distribution of galaxy merger histories we cannot ascertain how often such realistic disks are created. It has also become clear that a halo's merging history is related to its angular momentum (Maller et al. 2002; Vitvitska et al. 2002), and the way that baryons trace that merging history may play an important role in determining disk sizes (Maller \& Dekel 2002).

Our results for the merger statistics of SPH galaxies complement analytic and numerical calculations of dark halo merger statistics (Kauffmann \& White 1993; Lacev \& Cole 1993, 1994; Somerville \& Kolatt 1999; Somerville et al. 2000; Wechsler et al. 2002). They significantly improve on the earlier study of Murali et al. (2002) by using a higher resolution simulation and, equally important, by tracking merger histories of individual systems rather than globally averaged merger and accretion rates. We describe the simulation and our method of constructing merger trees in $\$ 2$ We present merger statistics in $\$ 3$ and in $\$ 4$ we use the merger histories to compute Hubble type distributions. We summarize our results and discuss directions for future work in $\$ 5$

\section{BUILDING MERGER TREES IN THE SIMULATION}

The simulation was evolved using PTreeSPH (Dave et al. 1997), a parallel version of the SPH code of Katz et al. (1996), for a flat $\Omega_{m}=0.4$ cosmology with $\sigma_{8}=0.8$, a Hubble constant $H_{0}=100 h \mathrm{~km} \mathrm{~s}^{-1} \mathrm{Mpc}^{-1}$ with $h=0.65$, a baryon content $\Omega_{b}=0.047$, and a spectral index $n=0.93$. Our choices of $\sigma_{8}, H_{0}, n$, and $\Omega_{b}$ are reasonably close to the latest cosmological parameters estimated from the CMB and large scale structure (Spergel et al. 2003; Eisenstein et al. 2005), while our value of $\Omega_{m}$ is higher by about $1.5 \sigma$. The box is $22.22 h^{-1} \mathrm{Mpc}$ on a side, with $128^{3}$ dark matter particles and $128^{3}$ gas particles. The limited size of the simulation could have a significant impact on our statistics - in particular there is only one cluster mass halo in our simulation volume, and that halo is itself anomalously large for a box of this size. The dark matter particle mass is $7.88 \times 10^{8} M_{\odot}$, and the gravitational softening is a $5 h^{-1}$ comoving $\mathrm{kpc} \mathrm{cu}-$

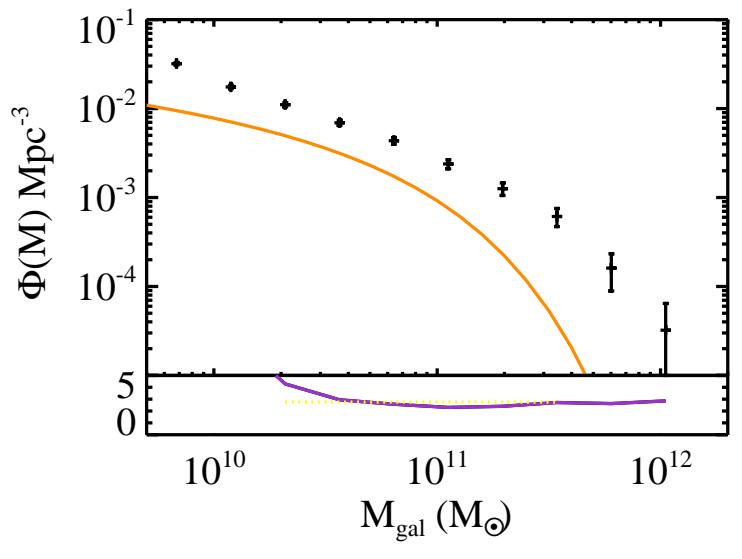

Figure 1. The upper panel shows the cumulative galaxy mass function at $z=0$ for our simulation and, for comparison, the Schechter function fit of Bell et al. (2003b). The y-axis is in comoving units with $h=0.7$ as we use throughout this paper. The simulations produce far too many low and high mass galaxies, while galaxies around the bend in the Schechter function are a factor of 2-3 too massive. The bottom panel shows the factor by which the simulated galaxies masses should be divided in order to agree with the observations. For the galaxy masses in which we are interested, $2 \times 10^{10} M_{\odot}<M_{\text {gal }}<6 \times 10^{11} M_{\odot}$, the correction is roughly a factor of 2.75 as shown by the dotted line, and it does not depend strongly on galaxy mass.

bic spline, roughly equivalent to a Plummer force softening of $3.5 h^{-1}$ comoving $\mathrm{kpc}$. The gas particles start off with masses of $1.06 \times 10^{8} M_{\odot}$, though this changes as star particles are created. This simulation has previously been used for studying quasar absorption systems (Gardner 2001), the X-ray properties of galaxy groups (Davé et al. 2002), the correlation between Lyman Break Galaxies and Ly $\alpha$ forest absorption (Kollmeier et al. 2005), and the histories and mechanisms of gas accretion (Murali et al. 2002; Kereš et al. 2005). Because the uncertainty in the Hubble parameter has been significantly reduced with recent observations and is now known to be quite close to $h=0.7$, we scale all observable quantities to the values appropriate for $h=0.7$ and do not quote explicit $h$ dependences. (For example, we take the box volume to be $22.22 / 0.7=31.75 \mathrm{Mpc}$ on a side.)

We analyze 227 output time steps between $z=8$ and $z=0$, spaced to give a time resolution better than 150 Myr. At each step, galaxies are identified using the Spline Kernel Interpolative DENMAX (SKID) ${ }^{1}$ algorithm (Gelb \& Bertschinger 1994; Katz et al. 1996). This algorithm consists of five basic steps: (1) the smoothed baryonic density field is determined; (2) baryonic particles are moved towards higher density along the initial gradient of the baryonic density field; (3) the initial group is defined to be the set of particles that aggregate at a particular density peak; (4) initial groups that are close together are then linked; (5) particles that do not satisfy a negative energy binding criterion relative to the group's center of mass are removed

${ }^{1} \mathrm{We}$ use the implementation of SKID by J. Stadel and T. Quin, which is publicly available at http://www-hpcc.astro.washington.edu/tools/skid.html 


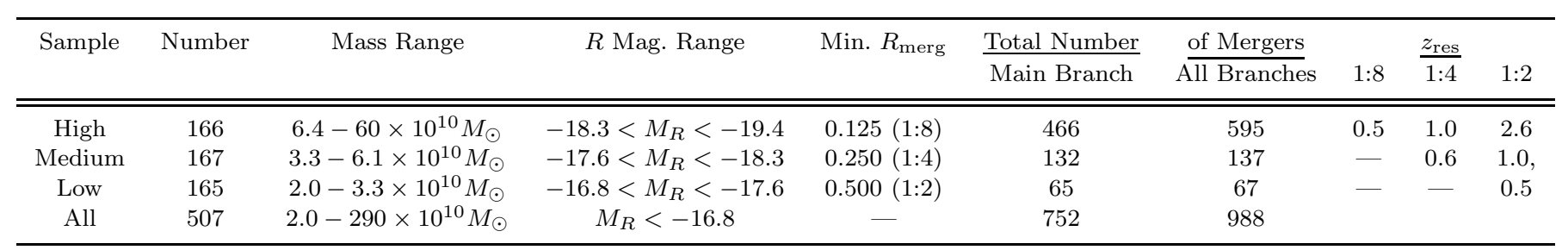

Table 1. The properties of galaxies in our three mass bins are shown as well as the properties of the sample as a whole. "All" includes 9 galaxies more massive then the upper mass cutoff of the high mass sample that are only used in $\S 3.2$ The $R$-band magnitude, corresponding to the same number density of objects in the Blanton et al. (2003) luminosity function is given for each mass bin. The minimum parent mass ratio for which the sample is complete and the total number of resolved mergers both along the main branch and for all branches are also given. For the medium and low mass samples essentially all resolved mergers are along the main branch. In the high mass sample about $30 \%$ of mergers occur off the main branch. We will focus on main branch mergers here as they are most important in determining the Hubble type of the $z=0$ galaxy.

from the group. These steps are applied to all star particles and to gas particles with temperatures $T<3 \times 10^{4} \mathrm{~K}$ and densities $\rho_{\text {gas }} / \bar{\rho}_{\text {gas }}>1000$. The resulting groups of cold gas and stars have masses and sizes similar to the luminous regions of observed galaxies (Katz 1992; Katz et al. 1996). The smallest systems reliably identified, as determined by comparison to higher resolution simulations of smaller volumes, have 64 particles, or a mass of $6.8 \times 10^{9} M_{\odot}$. However, for our merger study we restrict ourselves to galaxies with more than three times this mass, or $M_{\text {gal }}>2 \times 10^{10} M_{\odot}$ at $z=0$, since we also need to identify their smaller mass progenitors.

Previous merger trees that we are aware of have been built by identifying the progenitors (sometimes only the most massive progenitor) of each dark matter halo at time $t_{1}$ in a previous time step $t_{2}$, then repeating this procedure for all time steps (Lacev \& Cole 1994; Wechsler et al. 2002; Zhao et al. 2003; Lin et al.|2003; Peirani et al. 2004; Sheth \& Tormen 2004; Tormen et al. 2004). However, we have found a number of complications when trying to apply this algorithm to simulated galaxies. The main difficulty is in reliably defining a galaxy in each time step. As is sometimes the case with optical observations, it can be difficult to determine if merging systems should be identified as one or two galaxies, and on rare occasions a galaxy is not identified at all in one time step. If a galaxy's progenitor is missed in one time step, then the above algorithm will truncate the tree and find that a galaxy suddenly appears in the simulation. More commonly, we find that two galaxies have the same progenitor, so that galaxies appear to split and then re-form into one galaxy (see the top panel of Figure 2). A third complication is that particles can be stripped from both merger progenitors during the merging process but later join the merger remnant. Whether these particles should be counted as accreted mass or as part of the merger is again a matter of definition. These complications affect only a small minority of galaxies, and the application of SKID to the star and cold gas distributions generally yields robust galaxy identifications. However, with 227 time steps, even an algorithm that is reliable $99.9 \%$ of the time will encounter problems for one out of five merger trees.

We have developed a modified merger tree algorithm to address these issues. We distinguish between a SKID group, which is the output from running SKID on the simulation, and a galaxy, which we define in terms of our merger tree.
Usually the two are identical, but occasionally a galaxy can be made up of two or more SKID groups, or contain particles not in any SKID group, or both. We identify all SKID groups at $z=0$, trace each group's members back through all previous time steps, and identify all progenitor SKID groups containing at least 16 of these members. ${ }^{2}$ If a particle is not part of a SKID group in a particular time step but is part of a SKID group at an earlier time step, then we assign it to a "virtual" SKID group. These virtual assignments allow a SKID group to have multiple descendants, at least temporarily, usually during and after mergers.

To build the merger tree of a SKID group identified at $z=0$ (or another redshift chosen for the analysis), we trace its progenitors back in time but always combine all of the descendants (real and virtual) of a progenitor SKID group into a single object. This approach deals with the problem of temporarily split off particles or divided galaxies. We do not count particles that are not in "real" SKID groups at $z=0$. Occasionally the two descendants of a single galaxy never rejoin at a later time. When this occurs near $z=0$, we interpret it as an interrupted merger process and anticipate that the two descendants would rejoin if the simulation were run into the future. Therefore, when multiple descendants produce two branches reaching $z=0$, we combine them into one galaxy if the split occurred within 0.7 Gyr (half the dynamical time of the dark halo). If the two branches have remained separate longer than that, we assume that they will never rejoin, and instead separate the common progenitor into two galaxies, each one getting the descendant's fraction of the total mass of the SKID group. In these cases we always find that the SKID group has two parents with masses nearly identical to those of the two descendants; thus it appears that what has happened is that two galaxies have momentarily passed close enough to one another to be identified as one SKID group but do not have a strong physical interaction. The combining of unmerged descendants at $z=0$ means that we start off with 516 SKID groups that turn into 507 merger trees (galaxies).

As is generally the case in simulations of this sort, efficient cooling leads to galaxy masses at $z=0$ that are systematically larger than observational estimates. Figure 1

2 We have checked that our trees remain essentially unchanged if we change this requirement to 8 or 32 particles. 


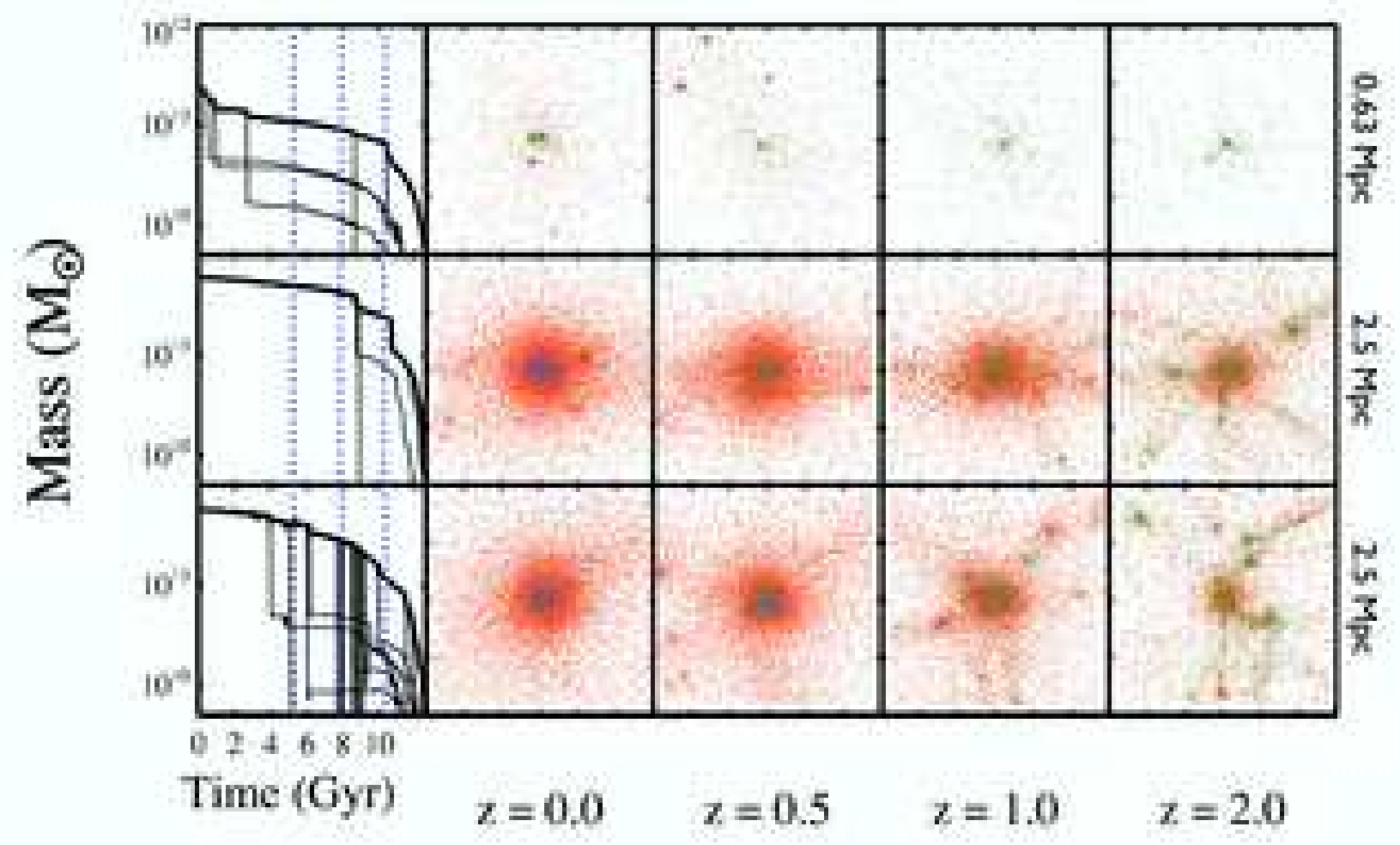

Figure 2. The figure shows the mass accretion histories for three example galaxies. In each row, the leftmost panel shows the masses of all of the galaxy's progenitors as a function of look backtime. When galaxies merge the lower mass progenitor is connected to the main progenitor by a vertical line. Vertical dotted lines mark redshifts $z=0.5,1$, and 2 . The remaining panels show projected particle distributions at $z=0,0.5,1$, and 2 , in regions 0.63 comoving Mpc (top row) and 2.5 comoving Mpc (middle and bottom rows) on a side. Green points denote particles that end up in the galaxy at $z=0$. Red and blue points mark other gas and star parciles, respectively.

shows the $z=0$ cumulative mass function, $\Phi\left(M_{\text {gal }}\right)$, of this simulation in comparison to the functional fit to the $z=0$ mass function estimate of Bell et al. (2003b), which uses galaxy luminosities and colors to infer stellar masses assuming a "diet Salpeter" initial mass function (IMF). The cumulative mass function is just the number density of objects with mass greater than $M$, or

$\Phi(M)=\int_{M}^{\infty} \phi\left(M^{\prime}\right) d M^{\prime}$

The simulated galaxies are too massive at all number densities, with the discrepancy worsening at low and high mass values. The source of this discrepancy remains unclear even after many years of study. It is possible that it is partly a numerical artifact (e.g. Springel \& Hernquist 2002), or the result of insufficient supernova (e.g. Dekel \& Silk 1986) or AGN (e.g. Omma et al. 2004) feedback in the simulations, or the result of incorrectly assuming a universal stellar IMF. Kereš et al. (2005) argue that a physical mechanism that suppresses "hot" accretion from shock heated gas halos would substantially reduce galaxy masses, especially above $M_{*}$. Such a mechanism might involve AGN feedback or conduction, or it might simply emerge from a proper treatment of multi-phase cooling (Maller \& Bullock 2004), which can reduce the overall rate of cooling onto massive galaxies (see Dekel \& Birnboim 2004, for a recent review of all these processes).

Because we compute merger statistics in terms of galaxy mass ratios, we are hopeful that any solution to the global mass discrepancy will have only a modest effect on the galaxy merger statistics that we present here. There may, however, be some changes to merger rates because lower mass galaxies have longer dynamical friction timescales. We plan to investigate the importance of this effect in future work. The correction to galaxy masses needed to make the simulated mass function agree with the observations is shown in the bottom panel of Figure 1 In the galaxy mass range that we consider, $2 \times 10^{10} M_{\odot}<M_{\text {gal }}<6 \times 10^{11} M_{\odot}$ at 


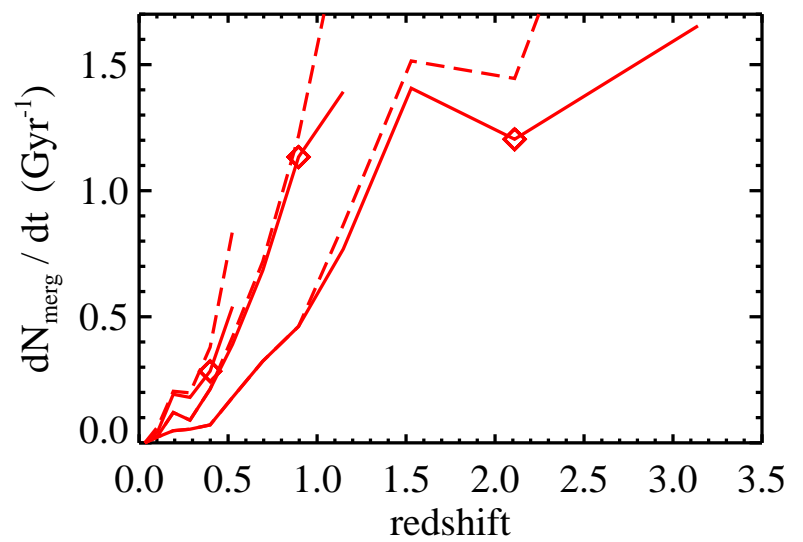

Figure 3. The number of mergers per galaxy as a function of redshift for the high mass sample. The three solid lines are, from top to bottom, $R_{\text {merg }}>0.125, R_{\text {merg }}>0.25$, and $R_{\text {merg }}>0.5$. The dashed lines show the maximum number of possible mergers including unresolved mergers. The diamonds mark the last bin where the Poisson error on the resolved mergers is larger than the possible contribution of unresolved mergers and the lines are continued for one bin past this point. The dividing redshift between these bins and the next are $z_{\text {res }}=0.5,1.0$ and 2.6.

$z=0$, the correction is well approximated by a constant factor of 2.75 (dotted line). Thus we expect that a physical or numerical fix to the mass discrepancy would have only modest impact on the merger rates at a given mass ratio. However, the correction becomes more significant at low masses (a factor of 18 for the lowest mass bin), which suggests that we may significantly overestimate the number of mergers when one parent has a mass less than $10^{10} M_{\odot}$. We will use the galaxy masses from the simulations in our discussion, but when connecting to observations we note that to get the correct number density of galaxies these masses should be divided roughly by a factor of 2.75 . An alternative way to make the connection to observed galaxy populations is to match the cumulative space density above mass thresholds in our simulation to the cumulative space density computed from the observed galaxy luminosity function Blanton et al. 2003), assuming that luminosity is a monotonic function of mass. The luminosities implied by this number density matching procedure are given in Table 1

\section{THE PROPERTIES OF GALAXY MERGER TREES}

Figure 2 illustrates the merger history of three simulated galaxies, selected to show a range of behaviors. In the left panels, lines show the baryonic masses (stars plus cold gas) of each galaxy's progenitors as a function of lookback time. In the second column, green points mark particles that are members of the galaxy at $z=0$, and blue points mark stars in other galaxies. Red points mark the surrounding gas particles, most of which are at high temperature. The remaining panels show the positions of these particles at $z=0.5,1$, and 2 , in regions of constant comoving size. Green points always represent particles that will be in the galaxy at $z=0$.

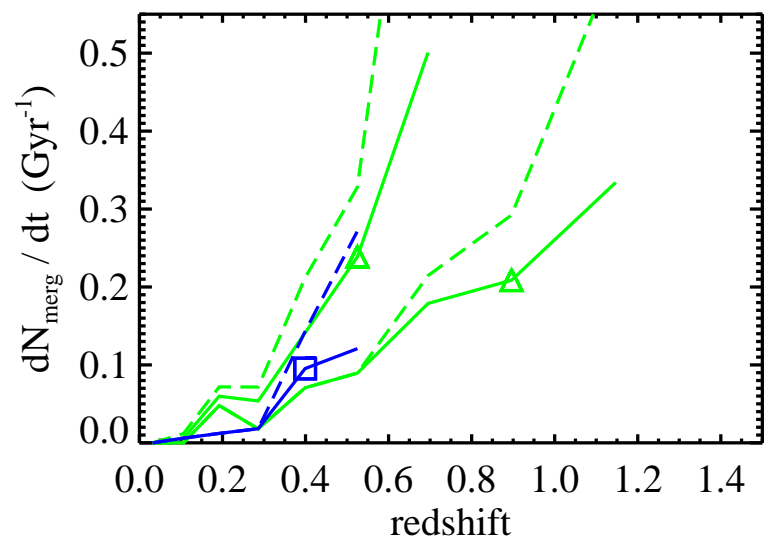

Figure 4. The number of mergers per galaxy as a function of redshift for the medium and low mass samples. The triangles denote the medium mass sample with the upper solid line being $R_{\text {merg }}>0.25$ and the lower solid line being $R_{\text {merg }}>0.5$, while the square marks the line for the low mass sample with $R_{\text {merg }}>0.5$. The dashed lines show the maximum number of possible mergers including unresolved mergers. The symbols mark the last bin where the Poisson error on the resolved mergers is larger than the possible contribution of unresolved mergers and the lines are continued for one bin past this point. The dividing redshift between these bins and the next are $z_{\text {res }}=0.5,0.6$ and 1.0 for the low mass sample and the medium mass sample with $R_{\text {merg }}>0.25$ and $R_{\text {merg }}>0.5$, respectively.

The topmost galaxy has three clearly identifiable progenitors at $z=0.5$. It experiences two major mergers (roughly 1:3) in the final Gyr, and it still has a bimodal appearance at $z=0$. In fact, SKID splits the system into two components at $z=0$, but they are a single component at an earlier output and are therefore counted as a single galaxy. The galaxy in the middle panels undergoes its last major merger at $z \sim 1.2$, and thereafter it grows by smooth accretion, doubling its mass by $z=0$. The morphological type recipe that we adopt in $\$ 4$ (similar to that in semi-analytic models) assigns the post-merger accretion to a disk component and therefore assigns this galaxy a bulge-to-total mass ratio of $\sim 0.5$. However, if some mechanism suppresses hot gas accretion at late times (Binnev 2004; Katz et al. 2003; Dekel \& Birnboim 2004; Kereš et al. 2005), then the bulge fraction would be higher (see $\$$ for further discussion). The galaxy in the bottom panel experiences many mergers, but all of them are minor; our morphological type recipe would therefore identify this as a disk-dominated system.

In quantitative terms, we would like to know the function $\Psi$, where

$\Psi\left(M_{\text {gal }}, z, R_{\text {merg }}\right)=\frac{\partial P_{\text {merg }}}{\partial t \partial M_{\text {gal }} \partial R_{\text {merg }}}$,

the probability that a galaxy with a mass between $M_{\text {gal }}$ and $M_{\text {gal }}+d M_{\text {gal }}$ at a redshift $z$ was the result of the merging of two galaxies with a mass ratio between $R_{\text {merg }}$ and $R_{\text {merg }}+d R_{\text {merg }}$ in the preceding time interval $d t$. We define $R_{\text {merg }}<1$, i.e., it is the ratio of the baryonic mass of the smaller parent to that of the larger parent. Unfortunately, we do not resolve a large enough dynamic range to 


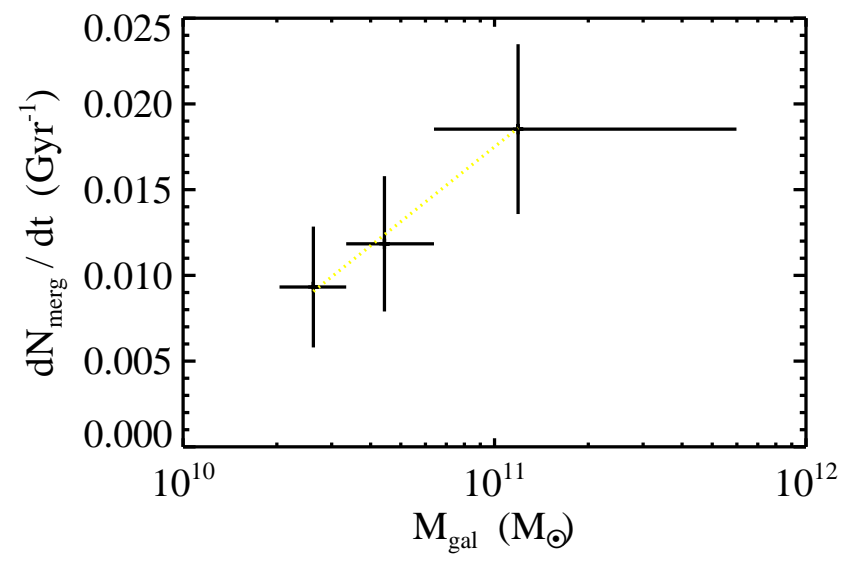

Figure 5. The number of nearly equal mass mergers $\left(R_{\text {merg }}>\right.$ $0.5)$ per galaxy, that occur for $0>z>0.5$ as a function of the galaxy mass $M_{\text {gal }}$ at $z=0$. The number of mergers is strongly dependant on the galaxy mass. The dashed line shows a linear fit to $\log \left(M_{\text {gal }}\right)$ with a slope of 0.0145 .

fully characterize the function $\Psi$, so instead we study several interesting "projections" of it to learn about its overall parameter dependence.

With the morphological assignment recipe of 4 galaxy's Hubble type is determined by the merger history of its "main" progenitor, the most massive progenitor at each output. If the Hubble types of the merging galaxies change the result of the merger, e.g. because bulges behave differently from disks, then the merger history of the minor progenitors also matters. However, this effect is likely to be of secondary importance, and in this paper we will not distinguish between mergers of disk and bulge galaxies. Table 1 lists the number of resolved main branch mergers (mergers involving a galaxy's main progenitor) and the total number of resolved mergers for all of our merger trees. Only for the high mass sample, $M_{\text {gal }}>6.4 \times 10^{10} M_{\odot}$, do we resolve a significant number of off-main branch mergers. In most cases, therefore, we present results only for main branch mergers, but we occasionally compare the statistics of main branch mergers to those of all mergers for the high mass sample.

\subsection{Galaxy-averaged merger statistics}

Figures 3 and 4 present our main characterizations of $\Psi\left(M_{\text {gal }}, z, R_{\text {merg }}\right)$ : the average number of mergers per galaxy per Gyr above $R_{\text {merg }}$ thresholds of $0.125,0.25$, and 0.5 for galaxies in the high mass sample (Fig. 3) and the medium and low mass samples (Fig. 4). The relation of $d N_{\text {merg }} / d t$ to $\Psi\left(M_{\text {gal }}, z, R_{\text {merg }}\right)$ is

$$
\begin{aligned}
\frac{d N_{\mathrm{merg}}}{d t} & =\frac{1}{N_{\text {gal }}} \sum_{i=1}^{N_{\text {gal }}} \int_{R_{\text {min }}}^{1} \Psi\left(M_{i}, z, R_{\text {merg }}\right) d R_{\text {merg }} \\
\approx & \frac{1}{\bar{n}} \int_{M_{\min }}^{M_{\max }} d M \frac{d n}{d M} \int_{R_{\min }}^{1} \Psi\left(M_{i}, z, R_{\text {merg }}\right) d R_{\text {merg }}
\end{aligned}
$$

where $R_{\text {min }}$ is the mass ratio threshold, $N_{\text {gal }}$ is the number of simulated galaxies in the mass range $M_{\min }$ to $M_{\max }, d n / d M$ is the galaxy baryonic mass function, and $\bar{n}$ is the mean space density of galaxies in the mass range. We compute $d N_{\text {merg }} / d t$ by counting mergers in a redshift interval $\Delta z$

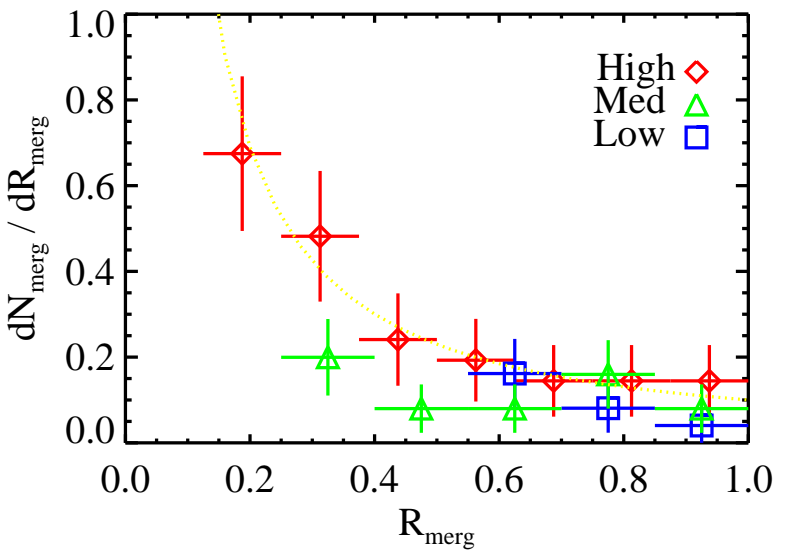

Figure 6. The fraction of mergers with parent mass ratios $R_{\text {merg }}$ for galaxies in the high (diamonds), medium (triangles) and low (square) mass samples are shown for mergers in the redshift range $0<z<0.5$. The points are normalized by the width of the bin. The distribution of the high mass sample is well fit by a function $\propto R_{\text {merg }}^{-1.2}$ plotted as a dotted line. For the medium and low mass samples we do not have enough data to constrain the shape of the distribution, so we do not know if this distribution is a function of galaxy mass or redshift.

and dividing by the corresponding time interval $\Delta t$, with typical values $\Delta t \approx 1 \mathrm{Gyr}$. The lower mass ratio mergers (i.e., $R_{\min }=1 / 8$ or $1 / 4$ ) can only be resolved for higher mass galaxies. Merger rates are substantially higher for massive galaxies - e.g., the average rate of $R_{\text {merg }}>0.5$ mergers at $z=0.3$ is $0.054 \mathrm{Gyr}^{-1}$ for the high mass sample and $0.018 \mathrm{Gyr}^{-1}$ for both the medium and low mass samples.

Our main limitation in computing these statistics is that we do not reliably resolve SKID groups with fewer than 64 particles. Thus, when a galaxy's mass increases by 63 particles we cannot tell without detailed examination whether this growth was the result of a merger or of smooth accretion. The directly calculated rates shown by solid lines in Figures 3 and 4 are therefore lower limits to the true rates. Dashed curves accompanying the solid curves show rates that include the maximum contribution of unresolved mergers, assigning all growth of up to $63 \mathrm{SPH}$ particle masses that is not in resolved mergers to unresolved mergers. At low redshift there are no unresolved mergers, but as we go back in redshift and the galaxy masses decrease, the number of possible unresolved mergers increases. We stop each line when the number of possible unresolved mergers is greater than the Poisson uncertainty in the number of resolved mergers. Symbols mark the last output bin before this limiting redshift, where we believe we resolve enough of the mergers to robustly study their properties. Since the assumption used for the dashed lines is extreme, we expect that the solid lines are generally closer to the true rates.

For the high mass sample, the limiting redshifts for $R_{\text {merg }}>0.125,0.25$ and 0.5 are $z_{\text {res }}=0.5,1.0$ and 2.6 , respectively. For medium masses, merger ratios of $R_{\text {merg }}>$ 0.25 and 0.5 can be resolved to $z_{\text {res }}=0.6$ and 1.0 , respectively. For the low mass sample, $R_{\text {merg }}>0.5$ can be resolved only to $z_{\text {res }}=0.5$. To compare all three samples, we must therefore restrict ourselves to $R_{\text {merg }}>0.5$ and $z \leq 0.5$. 
Figure 5]quantifies the mass dependence of merger rates seen in Figures 3 and 4 To maximize statistics, we count all mergers with $R_{\text {merg }}>0.5$ occurring between $z=0$ and $z=0.5$. The mean number of mergers per galaxy in this interval follows a best-fit relation

$N_{\text {merg }}=0.0145 \log _{10}\left(\frac{M_{\text {gal }}}{10^{11} M_{\odot}}\right)+0.0175$,

where $M_{\text {gal }}$ is the galaxy mass at $z=0$. We can therefore anticipate a strong dependence of Hubble type frequencies on galaxy mass. We find an even steeper best-fit slope for $R_{\text {merg }}>0.25$, but with only two points it is hard to draw meaningful conclusions from this difference.

Figure 6] shows the distribution of parent mass ratios for main branch mergers in the interval $0<z<0.5$. In the high mass sample, the number of mergers is proportional to $R_{\text {merg. }}^{-1.2}$. There are not enough bins in the low and medium mass samples to reliably infer a dependence (or lack thereof) on galaxy mass. The -1.2 slope for the high mass sample implies that high mass ratio mergers dominate the merger growth rate, since the average rate at which a galaxy gains mass by mergers above a threshold $R_{\min }$,

$\frac{d M}{d t} \propto \int_{R_{\min }}^{1} \Psi\left(R_{\mathrm{merg}}\right) R_{\mathrm{merg}} d R_{\mathrm{merg}} \propto 1-R_{\mathrm{min}}^{0.8}$,

has already reached half of its asymptotic value for $R_{\min }=$ 0.42 .

\subsection{Volume-averaged Merger Rates}

We now turn to the volume-averaged merger rate of galaxies (the mean number of mergers per comoving $\mathrm{Mpc}^{3}$ per Gyr), which can be estimated observationally by counting either recent merger remnants or close pairs that will merge in the near future, and dividing by an estimated merger timescale. The merger rate of all galaxies (not just the main progenitor branch) is shown in Figure $\mathbf{7}$ for mergers with $R_{\text {merg }}>0.5$. We plot the result for all branches because observations cannot determine which merger remnants will end up merging with other galaxies at future times. Dashed lines include the possible contribution of unresolved mergers. We continue curves past the limiting redshift $z_{\text {res }}$ adopted in Figures 3 and 4 because the range of possible merger rates is still an interesting prediction, even though the contribution of unresolved mergers may be significant. Although we are likely to be missing some mergers above $z_{\text {res }}$, we think that the resolved merger rates (solid lines) are probably closer to the true merger rate predictions than the dashed lines, which are upper limits based on extreme assumptions.

The naked error bars in Figure 7 show the observational estimates of Lin et al. (2004) derived from the DEEP2 survey (Davis et al. 2003), for galaxies with luminosities $-19<M_{B}<-21$. These points lie below our model predictions, especially at $z>1$. The luminosity range roughly corresponds to the mass range of our high mass sample, except that these are the galaxy luminosities at the observed redshift, not at $z=0$. If we restrict ourselves to galaxies that are in that mass range at the plotted redshift, then we get the dot-dash line with crosses in Figure 7 which agrees fairly well with the observations at $z<1$ but remains high by a factor $\sim 2$ at $z>1$. Evolution of stellar mass-to-light

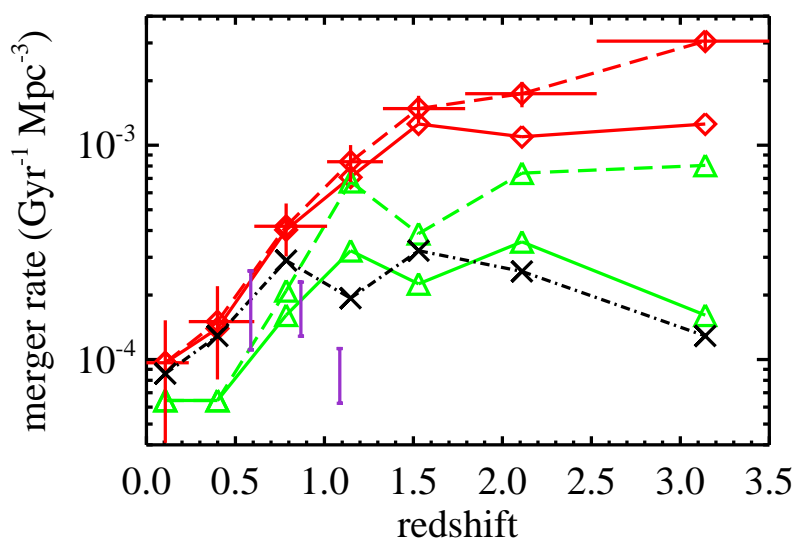

Figure 7. The number of mergers per Gyr per comoving $\mathrm{Mpc}^{3}$ is shown as a function of redshift. The high mass sample (diamonds) and medium mass sample (triangles) are shown for all resolved mergers (solid lines) and including the total possible number of unresolved mergers (dashed lines). We see that the contribution of unresolved mergers is negligible for $z<1.7$ in the high mass sample and $z<1.0$ in the medium mass sample. The merger rate when galaxies are selected by their mass at that redshift is shown as the dot-dash line with crosses. This is more relevant to what one sees observationally in a flux limited sample. For comparison the data of Lin et al. (2004) is shown as the naked error bars for a similar range in stellar mass. The error bars on the upper most points are representative of the errors for all the simulation points.

ratios changes the correspondence between luminosity and stellar mass, and the range of mass-to-light ratios becomes larger at earlier epochs, so a full assessment of this mild discrepancy will require more detailed modeling of the stellar populations of the simulated galaxies, and more detailed replication of the observational procedures for estimating merger rates. The systematic uncertainties in matching theoretical and observational samples are minimized if merger rates are given divided by the number density of objects being sampled, as in our Figures 3 and 4 When data are presented as in Figure 7 the number of mergers per galaxy is convolved with evolution of the number density of galaxies and of their properties, making it more difficult to isolate the source of discrepancies.

Figure 8 shows the distribution of the total number of mergers a galaxy undergoes during its history, for different mass samples and mass ratio thresholds. Figure legends list the average number of mergers per galaxy for each mass bin and parent mass ratio. Curves in each panel show a Poisson distribution with the corresponding mean, and in all cases they describe the measured distribution with reasonable accuracy. This result suggests that each merger is an independent event; the average probability of mergers depends on galaxy mass, but in a given mass range there are not "merger heavy" or "merger light" galaxies beyond what is expected from Poisson statistics. 


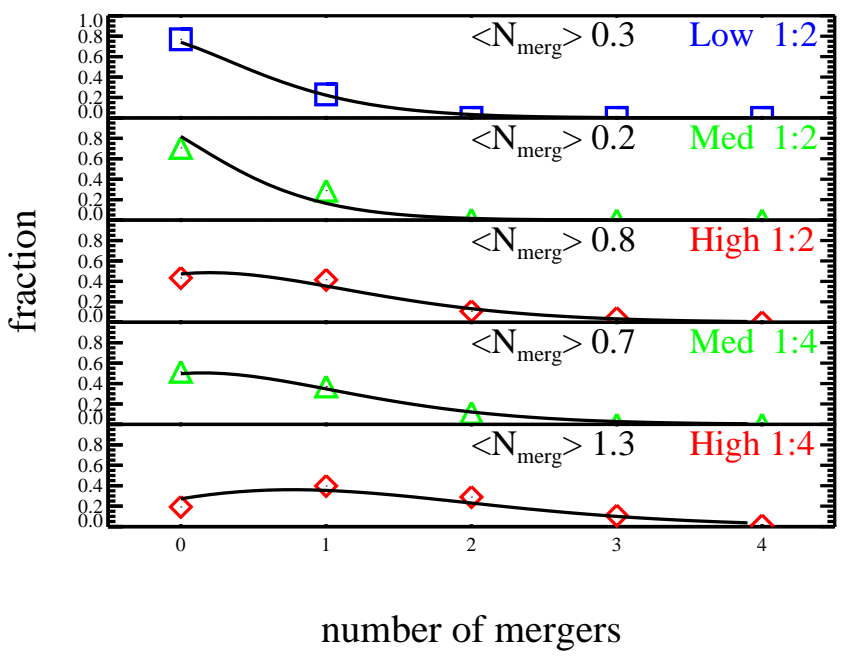

Figure 8. The number of mergers a galaxy undergoes for the low, medium and high mass samples and for parent mass ratios of $1: 2$ and $1: 4$. We see that all five are well fit by a Poisson distribution with a galaxy having a mean number of mergers of $0.3,0.2,0.8,0.7$ and 1.3 , respectively.

\subsection{Last Major Merger}

We now turn to another interesting characteristic of merger histories, the distribution of redshifts at which galaxies experience their last major merger. Figure 9] plots the fraction of galaxies that have undergone a merger above a $1: 2$ or $1: 4$ mass threshold since redshift $z$, in the three mass bins. Including unresolved mergers has only a modest effect on this result and is most significant at higher redshifts (see dashed lines). We see that in all cases a sizeable fraction of galaxies have never undergone a merger since the time the galaxy first crossed our mass resolution threshold of $6.8 \times 10^{9} M_{\odot}$. (Galaxies in the low mass sample have grown by a factor of $3-5$ since that time, while galaxies in the high mass sample have grown by at least a factor of nine.) In the medium mass sample, for example, only half of the galaxies experienced a merger with mass ratio larger than 1:4, and less than $30 \%$ had a 1:2 merger.

The symbols in Figure 9 mark the median redshift of the most recent major merger for those galaxies that did experience a merger. About half of these mergers occur at $z \lesssim 1-1.5$. We can compare this result to the predictions for dark matter halos. Wechslen (2001), Fig. 137, shows the redshift distribution of the last 1:3 merger for dark matter halos in differing mass ranges. If we simply approximate the mass of the halo for each of the $z=0$ galaxies by multiplying by $2 \Omega_{m} / \Omega_{b}$, we obtain median halo masses of $0.6,1$ and $2 \times$ $10^{12} M_{\odot}$ for the low, medium and high mass galaxy samples, respectively. ${ }^{3}$ Wechsler (2001) finds that $40 \%$ and $28 \%$ of halos with masses of 0.6 and $1.4 \times 10^{12} M_{\odot}$, respectively, have not had a major merger (greater than 1:3) since $z=$ 4. For those halos that did have major mergers at $z<4$, the median redshift of the most recent merger is $z=1$ in

3 For this halo mass range, we find in the simulation that roughly $50 \%$ of the halo baryons end up in the central galaxy.

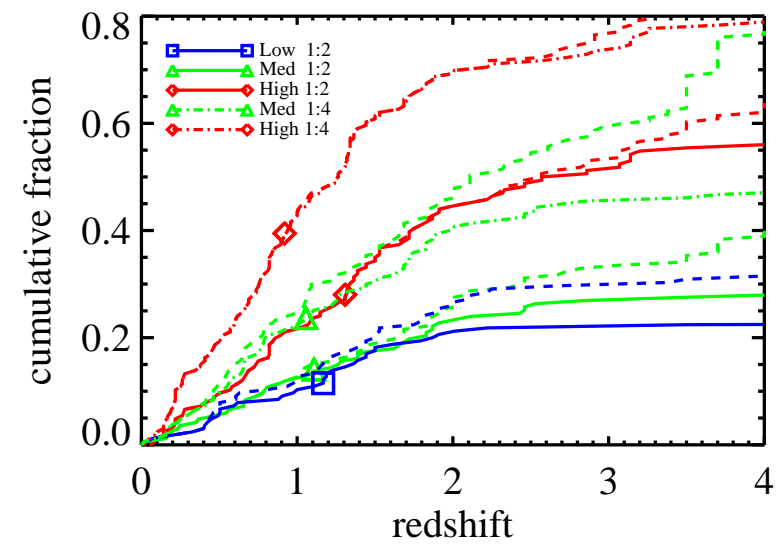

Figure 9. The fraction of galaxies that have undergone a merger of parent mass ratio $R_{\text {merg }}>0.5$ (solid lines) or $R_{\text {merg }}>0.25$ (dot-dash lines) as a function of redshift is shown for the three mass samples: high (diamonds), medium (triangles) and low (squares). We see that in all cases some galaxies never undergo a merger of that parent mass ratio. The corresponding dashed lines show the cumulative fraction including unresolved mergers. The median redshift of galaxies that do undergo a merger is marked by the symbols.

both cases. Compared to the dark halos, more galaxies in our simulation are merger free, and the median redshift of the last major merger is slightly higher. However, Wechsler (2001) uses a slightly different cosmological model $\left(\Omega_{m}=\right.$ $0.3, \Lambda=0.7, \sigma_{8}=1$, and $n=1$ ), which could plausibly account for differences of this magnitude.

The similarity of our galaxy merger rates to the Wechsler (2001) halo merger rates is broadly consistent with the idea that most major mergers of halos are quickly followed by mergers of their central galaxies. In future work we intend to perform a more detailed analysis of this point using halos identified in our simulation to investigate the relationship between dark halo and galaxy mergers. Unfortunately, a comparison of our results to semi-analytic models of galaxy formation is difficult, since most authors only present results that are meant to be compared directly with observations and do not present the intermediate results necessary for a comparison to the simulations or to other semi-analytic models. The only semi-analytic prediction with which we can compare is that of Baugh et al. (1996), who show plots similar to Figure 9] In their model, nearly all galaxies have had a major merger since $z \sim 2$. However, they consider an $\Omega_{m}=1$ cosmology, which is undoubtedly the major cause of this large difference from our results. A comparison of Figure 9 to semi-analytic predictions using the same cosmology would be interesting.

\section{THE DISTRIBUTION OF HUBBLE TYPES}

We now turn to determining a galaxy's Hubble type from its merger history. Simulations have shown that collisions between disk galaxies typically result in spheroidal systems (Toomre \& Toomre 1972; Barnes 1988; 
Hernquist 1992; Barnes \& Hernquist 1996; Naab et al. 1999; Naab \& Burkert 2003). We investigate a simple ansatz for the effect of mergers on morphology: if the mass ratio $R_{\text {merg }}$ exceeds a threshold $R_{\text {major }}$, we convert the entire baryonic mass of the merger remnant to a spheroid. ${ }^{4}$ Mergers below the $R_{\text {major }}$ threshold have no effect. All mass accreted after the last major merger is assigned to the disk component.

Figure 10] shows the distribution of bulge-to-total mass ratios computed using this recipe and the simulated merger histories, for the three mass bins and $R_{\text {major }}$ thresholds of 1:2 and 1:4. At low bulge-to-total ratio, unresolved mergers could have a significant impact. At the low end of our mass range we only resolve the later two thirds of a galaxies mass accretion history, so up to one-third of its mass could have had an unresolved merger before we start resolving it. We therefore show, in addition to our predictions using resolved mergers, a prediction using the extreme assumption that each galaxy has the maximum possible contribution from unresolved mergers. In general, this maximal contribution simply shifts galaxies from the lowest bulge-to-total bin $(<0.1)$ to the next bin $(0.1-0.2)$. Thus, our prediction for the fraction of truly bulgeless low mass galaxies can be significantly affected by unresolved mergers, but our other predictions cannot.

For bulge-to-total ratios $>0.2$, the predicted distributions in Figure 10 are strikingly flat. In particular, the simulation produces relatively few systems that are close to $100 \%$ bulge, because after mergers galaxies continue to accrete gas and form new stars in a disk component in the simulation. As discussed by Kereš et al. (2005; see also Binnev 2004; Croton et al. 2005; Dekel \& Birnboim 2004), a mechanism that halts late-time accretion onto high mass galaxies, such as AGN feedback (Binnev \& Tabor 1995) or multi-phase cooling (Maller \& Bullock 2004) would improve the agreement between the simulations and observations by predicting lower masses and older stellar populations at the high end of the galaxy luminosity function. Reproducing the correct distribution of bulge-to-disk ratios could be a powerful test of such mechanisms, since the fraction of bulgedominated systems will change depending on the conditions assumed for shutoff of gas accretion.

As expected, the higher merger rates of massive galaxies lead to a greater frequency of high bulge-to-disk ratios in our highest mass bin. To summarize the mass dependence and compare to observations, we separate the galaxy populations into "early" and "late" subsets at a bulge mass fraction of 0.5 . For a major merger threshold of 1:2, the late-type fraction is about $85 \%$ in the low and intermediate mass bins but only $74 \%$ in the high mass bin. For a threshold of 1:4, the late-type fraction is $69 \%$ in the intermediate mass bin and $46 \%$ in the high mass bin; we cannot resolve 1:4 mergers in the low mass bin.

Figure 11 compares these results to an estimate of the

\footnotetext{
4 We have also investigated the recipe used in some semi-analytic models where for each merger of mass ratio $R_{\text {merg }}>R_{\text {major }}$ twice the mass of the less massive progenitor is converted into bulge, and this is done for all mergers along the galaxy's main branch. Final bulge-to-disk ratios for this "partial conversion" recipe are slightly lower than those for the "total conversion" recipe, but the qualitative results are very similar, so we do not present them separately.
}

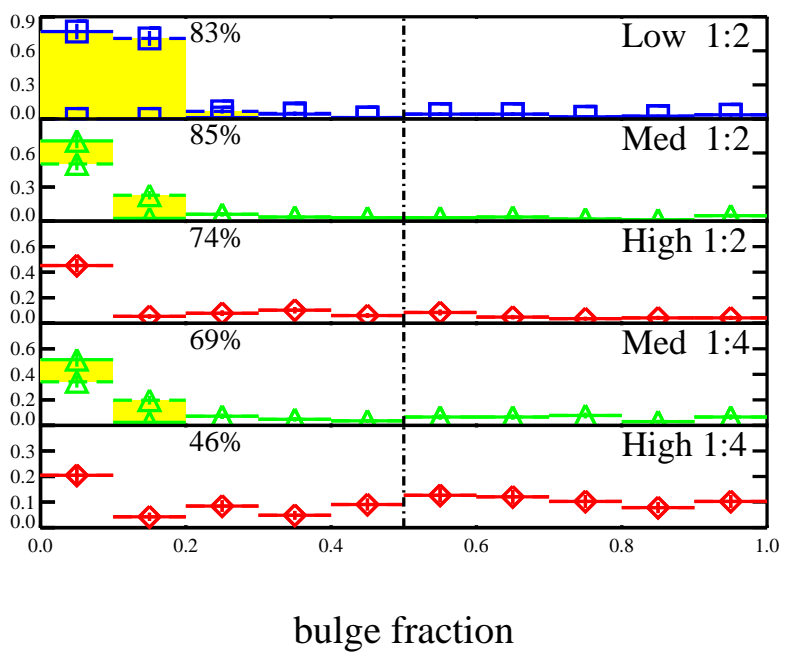

Figure 10. The bulge-to-total fraction is shown for each mass sample and for two choices of what constitutes a major merger, $R_{\text {major }}=0.5$ and $R_{\text {major }}=0.25$. The solid line includes only resolved mergers and the dashed line includes all possible mergers. The shaded region shows the range that could exist given our finite resolution. Dividing early and late type galaxies at a bulgeto-total ratio of 0.5 , we also indicate the late galaxy fraction, which is the same whether or not unresolved mergers are included.

early-type fraction derived from Bell et al.'s (2003b) determination of the early and late type stellar mass functions, using the 2MASS data set (Skrutskie et al. 1997; Maller et al. 2005). Because of the offset between predicted and observed galaxy mass functions discussed in $\$ 2$ we divide the simulated galaxy masses by 2.75 for this comparison; we are thus comparing populations of similar space density. For $R_{\text {major }}=1: 2$, the predicted early-type fraction is substantially below the observational estimate. For $R_{\text {major }}=1: 4$, the agreement is excellent, albeit with only two theoretical data points. We have checked that ratios of $1: 3$ and 1:5 are also in fairly good agreement. Bell et al. (2003b) determine galaxy types from concentrations, and the principal systematic uncertainty in this comparison is that we do not know how well their definition corresponds to our theoretical criterion $f_{\text {bulge }}=0.5$. The full distribution of bulgeto-disk ratios would be a powerful test of our predictions and, more generally, a powerful diagnostic of the physical processes that produce galaxy bulges. Extracting unbiased estimates of this distribution for large, well defined galaxy samples is a challenging task, but within reach of modern data sets (e.g. McIntosh et al. 2002).

\section{CONCLUSIONS}

We have used an SPH simulation of a $\Lambda$ CDM universe to calculate the statistics of galaxy merger histories. Our principal findings are as follows:

1. The incidence of major mergers is much higher for high mass galaxies. At $z=0.3$, we find a rate of 0.054 mergers per Gyr above a 1:2 mass threshold for galaxies with $M_{\text {gal }}>6.4 \times 10^{10} M_{\odot}$, but the rate for our lower mass galaxy samples is three times lower. 


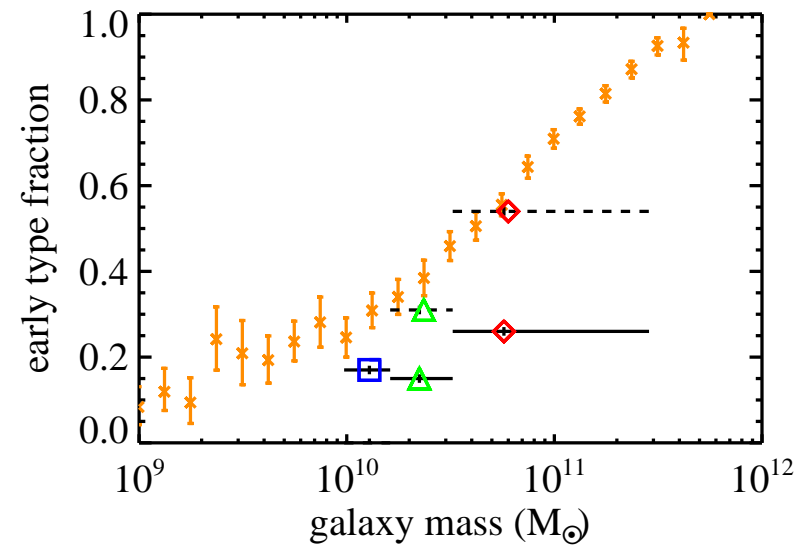

Figure 11. The fraction of galaxies that are early type as a function of the galaxies mass is shown for parent mass ratios of 1:2 (solid line) and 1:4 (dashed line). The crosses with error bars are the observed fraction derived from Bell et al. (2003b). The masses of the simulated galaxies have been divided by a factor of 2.75 to bring them into agreement with the observed stellar mass function.

2. The distribution of merger mass ratios is $N \propto R_{\text {merg }}^{-1.2}$ in our high mass sample (the only one for which our statistics are sufficient to make the estimate). With this logarithmic slope, major mergers dominate the total mass growth from mergers, with half of the mass coming in mergers above a mass ratio threshold $R_{\text {merg }}=0.4$. However, as shown by Murali et al. (2002) and Kereš et al. (2005), even the high mass galaxies in our simulations gain most of their mass through smooth gas accretion rather than mergers with preexisting galaxies. Our derived mass ratio distribution reinforces their arguments that the dominance of smooth accretion over mergers is not an artifact of limited numerical resolution.

3. For each mass and mass ratio bin, the merger rate climbs rapidly with redshift, roughly doubling between $z \sim 0$ and $z \sim 0.6$. The predicted merger rates are in reasonable agreement with observational estimates, but the systematic uncertainties in the comparison are large because of the difficulty of matching simulated and observed galaxy populations.

4. In each bin of mass and mass ratio, the number of mergers per galaxy is Poisson distributed with respect to the mean number for the bin. This suggests that each merger is, effectively, an independent event.

5. A substantial fraction of galaxies experience no major mergers after $z=1$, and many have no major mergers over the entire time that they are resolved in our simulations. For example, even in our high mass sample, only $45 \%$ of galaxies have an $R_{\text {merg }}>1: 4$ merger at $z<1$, and only $20 \%$ have an $R_{\text {merg }}>1: 2$ merger. For those galaxies that do experience major mergers, the median redshift of the most recent event is $z \sim 1-1.5$ in all mass and $R_{\text {merg }}$ bins.

The high frequency of quiescent merger histories is perhaps our most important result. If major mergers are indeed the primary mechanism of spheroid formation, then this large population of merger-free galaxies shows that the $\Lambda \mathrm{CDM}$ model can easily produce an acceptable fraction of late-type, nearly bulgeless systems. The predominance of quiescent merger histories is also good news for the galaxy angular momentum problem, since low redshift mergers are frequently responsible for removing angular momentum and shrinking disk sizes in simulations that focus on the formation of individual galaxies.

We have combined our merger histories with a simple, semi-analytic style recipe for spheroid formation to estimate bulge fractions of our simulated galaxies. With a bulge conversion threshold of 1:4, we find acceptable agreement with the observed trend of early-type galaxy fraction with galaxy mass, once we rescale the simulated galaxy masses to match the observed galaxy mass function. However, since most galaxies have continuing gas accretion after their last major merger, the fraction of truly bulgedominated systems (i.e., $f_{\text {bulge }} \gtrsim 0.8$ ) is small, even at high masses. This result suggests that some mechanism that shuts off gas accretion in high mass halos is needed to explain the observed distribution of galaxy morphologies. Many authors have already argued that a mechanism of this sort is required to reproduce the observed exponential cutoff of the galaxy luminosity function and the red colors of the most massive galaxies (e.g., Kauffmann et al. (1993); Benson et al. (2003); Binnev (2004); Croton et al. (2005); Dekel \& Birnboim (2004); Kereš et al. (2005)).

Our simulation volume is too small to allow a detailed investigation of the morphology-density relation. However, in the one cluster mass halo $\left(M_{\mathrm{vir}}=3 \times 10^{14} M_{\odot}\right)$ halo that forms in our simulation, we do not find the high fraction of early-type galaxies seen in observed clusters. This discrepancy suggests that some mechanism other than major mergers must contribute to morphological transformations in the cluster environment, such as ram pressure stripping Gunn \& Gott 1972) or "harassment" by weak perturbations (Moore et al. (1996).

We hope to extend this work in future studies that use simulations of larger dynamic range. These will allow us to investigate the correlation of merger histories with environment and to better characterize the merger histories of lower mass galaxies. We will also investigate the relationship between individual galaxy and dark halo merging histories, and we will check whether mechanisms that reduce galaxy baryon masses, such as galactic winds and suppression of hot accretion, also change the statistics of galaxy mergers. Comparison of our results to observations already suggests that the simplest picture of spheroid formation by mergers is not the full story. Future comparisons drawing on better simulations and more detailed observational analyses should yield much greater insight into the physics that determines galaxy morphologies.

\section{ACKNOWLEDGMENTS}

We thank Risa Wechsler and Mark Fardal for comments on an earlier draft of this paper. This project was supported by NASA ATP grant NAG5-13308, NASA LTSA grant NAG513102, and NSF grant AST-0205969. 
Galaxy Merger Statistics and Inferred Bulge-to-Disk Ratios in Cosmological SPH Simulations

\section{REFERENCES}

Barnes J. E., 1988, ApJ, 331, 699

Barnes J. E., Hernquist L., 1996, ApJ, 471, 115

Baugh C. M., Cole S., Frenk C. S., 1996, MNRAS, 282, L27

Bell E. F., Baugh C. M., Cole S., Frenk C. S., Lacey C. G., 2003, MNRAS, 343, 367

Bell E. F., McIntosh D. H., Katz N., Weinberg M. D., 2003, ApJS, 149, 289

Bendo G. J., Barnes J. E., 2000, MNRAS, 316, 315

Benson A. J., Bower R. G., Frenk C. S., Lacey C. G., Baugh C. M., Cole S., 2003, ApJ, 599, 38

Binney J., 2004, MNRAS, 347, 1093

Binney J., Tabor G., 1995, MNRAS, 276, 663

Blanton M. R., et al., 2003, ApJ, 592, 819

Cole S., Lacey C. G., Baugh C. M., Frenk C. S., 2000, MNRAS, 319, 168

Cox T. J., Primack J., Jonsson P., Somerville R. S., 2004, ApJL, 607, L87

Cretton N., Naab T., Rix H., Burkert A., 2001, ApJ, 554, 291

Croton D. J., et al., 2005, submitted to MNRAS, astro$\mathrm{ph} / 0508046$

Davé R., Katz N., Weinberg D. H., 2002, ApJ, 579, 23

Dave R., Dubinski J., Hernquist L., 1997, New Astronomy, 2,277

Davis M., et al., 2003, in Discoveries and Research Prospects from 6- to 10-Meter-Class Telescopes II. Edited by Guhathakurta, Puragra. Proceedings of the SPIE, Volume 4834, pp. 161-172

Dekel A., Birnboim Y., 2004, astro-ph/0412300

Dekel A., Silk J., 1986, ApJ, 303, 39

Eisenstein D. J., et al., 2005, astro-ph/0501171

Gardner J. P., 2001, ApJ, 557, 616

Gelb J. M., Bertschinger E., 1994, ApJ, 436, 467

Governato F., et al., 2004, ApJ, 607, 688

Gunn J. E., Gott J. R. I., 1972, ApJ, 176, 1

Hatton S., Devriendt J. E. G., Ninin S., Bouchet F. R.,

Guiderdoni B., Vibert D., 2003, MNRAS, 343, 75

Hernquist L., 1992, ApJ, 400, 460

Kang X., Jing Y. P., Mo H. J., Boerner G., 2004, astro$\mathrm{ph} / 0408475$

Katz N., 1992, ApJ, 391, 502

Katz N., Keres D., Dave R., Weinberg D. H., 2003, in ASSL Vol. 281: The IGM/Galaxy Connection. p. 185

Katz N., Weinberg D. H., Hernquist L., 1996, ApJS, 105, 19

Kauffmann G., 1996, MNRAS, 281, 487

Kauffmann G., White S. D. M., 1993, MNRAS, 261, 921

Kauffmann G., White S. D. M., Guiderdoni B., 1993, MNRAS, 264, 201

Kereš D., Katz N., Weinberg D. H., Davé R., 2005,MNRAS, in press, astro-ph/0407095

Kollmeier J. A., Miralda-Escude J., Cen R., Ostriker J. P., 2005

Lacey C., Cole S., 1993, MNRAS, 262, 627

Lacey C., Cole S., 1994, MNRAS, 271, 676

Lin L., et al., 2004, ApJL, 617, L9

Lin W. P., Jing Y. P., Lin L., 2003, MNRAS, 344, 1327

Malin D. F., Carter D., 1983, ApJ, 274, 534

Maller A. H., Bullock J. S., 2004, MNRAS, 355, 694

Maller A. H., Dekel A., 2002, MNRAS, 335, 487
Maller A. H., Dekel A., Somerville R., 2002, MNRAS, 329, 423

Maller A. H., McIntosh D. H., Katz N., Weinberg M. D., 2005, ApJ, 619, 147

McIntosh D. H., Maller A. H., Katz N., Weinberg M. D., 2002, in Galaxy evolution, theory and observations

Moore B., Katz N., Lake G., Dressler A., Oemler A., 1996, Nature, 379, 613

Murali C., Katz N., Hernquist L., Weinberg D. H., Davé R., 2002, ApJ, 571, 1

Naab T., Burkert A., 2003, ApJ, 597, 893

Naab T., Burkert A., Hernquist L., 1999, ApJL, 523, L133

Nagashima M., Yoshii Y., 2004, ApJ, 610, 23

Navarro J. F., Steinmetz M., 2000, ApJ, 538, 477

Omma H., Binney J., Bryan G., Slyz A., 2004, MNRAS, 348, 1105

Peirani S., Mohayaee R., de Freitas Pacheco J. A., 2004, MNRAS, 348, 921

Robertson B., Yoshida N., Springel V., Hernquist L., 2004, ApJ, 606, 32

Schweizer F., Seitzer P., Faber S. M., Burstein D., Dalle Ore C. M., Gonzalez J. J., 1990, ApJL, 364, L33

Sheth R. K., Tormen G., 2004, MNRAS, 349, 1464

Skrutskie M. F., et al., 1997, in et al. F. G., ed., ASSL Vol. 210: The Impact of Large Scale Near-IR Sky Surveys, Kluwer Academic Publishing, Dordrecht, p 25

Somerville R. S., Kolatt T. S., 1999, MNRAS, 305, 1

Somerville R. S., Lemson G., Kolatt T. S., Dekel A., 2000, MNRAS, 316, 479

Somerville R. S., Primack J. R., 1999, MNRAS, 310, 1087

Spergel D. N., et al., 2003, ApJS, 148, 175

Springel V., 2000, MNRAS, 312, 859

Springel V., Hernquist L., 2002, MNRAS, 333, 649

Springel V., Hernquist L., 2004, astro-ph/0411379

Toomre A., Toomre J., 1972, ApJ, 178, 623

Tormen G., Moscardini L., Yoshida N., 2004, MNRAS, 350, 1397

Toth G., Ostriker J. P., 1992, ApJ, 389, 5

van Dokkum P. G., 2005, ApJ

Vitvitska M., Klypin A. A., Kravtsov A. V., Wechsler R. H., Primack J. R., Bullock J. S., 2002, ApJ, 581, 799

Wechsler R. H., 2001, PhD thesis, UCSC

Wechsler R. H., Bullock J. S., Primack J. R., Kravtsov A. V., Dekel A., 2002, ApJ, 568, 52

Zhao D. H., Mo H. J., Jing Y. P., Börner G., 2003, MNRAS, 339,12 